\title{
LABORATORY SPECTRA OF AMORPHOUS AND CRYSTALLINE OLIVINE: AN APPLICATION TO COMET HALLEY IR SPECTRUM
}

\author{
A. BLANCO, V. OROFINO \\ Department of Physics, University of Lecce, \\ C.P. 193, 73100 Lecce, Italy \\ E. BUSSOLETTI, S. FONTI \\ Institute of Experimental Physics, IUN, \\ Via Acton 38, 80133 Napoli, Italy \\ L. COLANGELI \\ Engineering Faculty, University of Cassino, \\ Via Zamosch 43,03043 Cassino, Italy \\ J. R. STEPHENS \\ Los Alamos National Laboratory, \\ Los Alamos, NM 87545, USA
}

\begin{abstract}
Among the various silicates proposed as components of cosmic dust grains, olivine is considered one of the most likely materials. In this work we present the infrared spectra of three different types of olivine grains: crystalline, amorphous and synthetic (also amorphous). While the first and second sample derive from the same natural mineral, the third one has been prepared in the laboratory according to the relative cosmic abundances of the elements. The experimental data are used to fit the emission feature observed in the comet Halley spectrum between 8 and $13 \mu \mathrm{m}$. Satisfactory results are obtained by using synthetic olivine mixed with a small amount (5\%) of crystalline grains.
\end{abstract}

\section{Introduction}

Silicates have been identified as components of the interstellar and interplanetary dust on the basis of agreement in shape and position of observed bands near 10 and $20 \mu m$ (for a recent review see Knacke, 1989).

The IR spectra of glassy silicates seem to provide a closer match to astronomical observations than crystalline minerals (Knacke, 1989; Kratschmer and Huffman, 1979). We have therefore undertaken a research program to measure the optical properties of glassy silicates having composition similar to minerals predicted in theoretical condensation sequences (Stephens et al., 1989). Among the samples examined one of the most "popular" is olivine which has also been recently proposed as an important component of cometary dust (Bregman et al., 1987; Campins and Ryan, 1989). In this work we fit the broad emission feature present in the comet Halley spectrum between 8 and $13 \mu m$ by using laboratory spectral data of natural (both glassy and crystalline) and synthetic amorphous olivine with

A.C. Levasseur-Regourd and H. Hasegawa (eds.), Origin and Evolution of Interplanetary Dust, 125-128.

- 1991 Kluwer Academic Publishers, Printed in Japan. 
chemical composition reflecting the cosmic abundances of the various components.

\section{Sample preparation and IR spectra}

The crystalline samples for IR spectroscopy have been prepared by grinding the natural minerals and embedding the resulting submicron particles in $\mathrm{KBr}$ pellets. Amorphous samples have been obtained by vaporizing the same materials by means of a LAMBDA PHYSIK LPX 315i excimer laser operating at a wavelength of $308 \mathrm{~nm}$. The repetition rate was $9 \mathrm{~Hz}$ with an energy of $400 \mathrm{~mJ} /$ pulse. The condensed smokes were directly collected on KRS-5 substrates inside a sample chamber which was filled with $\mathrm{O}_{2}$ at a pressure of 10 mbar (this work) or 1 atm (Stephens, 1980). No significant changes were, however, detected by varying the gas in the chamber.

We prepared synthetic samples because available olivine crystals, although they have a metal to silicon ratio very close to that found in typical O-rich stars, exhibit a ratio $\mathrm{Mg} / \mathrm{Fe}=9$. This value is about one order of magnitude larger than the cosmic one, and the composition (including the metal atoms) and the metal to silicon ratio are among the most important factors affecting the IR spectra. We produced and analyzed two synthetic olivine-type silicates with the appropriate cosmic elemental content:

$$
\left(\mathrm{Fe}_{0.6} \mathrm{Mg}_{0.4}\right)_{2} \mathrm{SiO}_{4} \quad \text { and } \quad\left(\mathrm{Fe}_{0.6} \mathrm{Mg}_{0.4} \mathrm{Al}_{0.035} \mathrm{Ca}_{0.03} \mathrm{Na}_{0.025}\right)_{2} \mathrm{SiO}_{4}
$$

In spite of the presence of $\mathrm{Al}, \mathrm{Ca}$ and $\mathrm{Na}$ in the second sample, the IR spectra of the two materials are essentially coincident. The targets for the laser were prepared starting from the following reagent grade materials:

$\begin{array}{lll}\text { Element } & \text { Starting Compound } & \text { Final Compound } \\ \mathrm{Mg} & \mathrm{MgCO}_{3} & \mathrm{MgO} \\ \mathrm{Fe} & \mathrm{Fe}_{2} \mathrm{O}_{3} & \mathrm{Fe}_{2} \mathrm{O}_{3} \\ \mathrm{Si} & \mathrm{SilicicAcid}_{\mathrm{Ca}} & \mathrm{SiO}_{2} \\ \mathrm{Na} & \mathrm{CaCO}_{3} & \mathrm{CaO} \\ \mathrm{Al} & \mathrm{Na}_{2} \mathrm{CO}_{3} & \mathrm{Na}_{2} \mathrm{CO}_{3} \\ & \mathrm{Gamma}-\mathrm{Al}_{2} \mathrm{O}_{3} & \mathrm{Gamma}-\mathrm{Al}_{2} \mathrm{O}_{3}\end{array}$

Each material was heated in successive steps up to $1000{ }^{\circ} \mathrm{C}$ to produce the needed oxides. $\mathrm{Na}_{2} \mathrm{CO}_{3}$ was not heated, and $\mathrm{Fe}_{2} \mathrm{O}_{3}$ and $\mathrm{Al}_{2} \mathrm{O}_{3}$ were heated just to dry the materials. Mixtures of powders for the synthetic samples were pressed in air (10 ton). Each pellet was then heated in a radio frequency furnace in an Ar atmosphere to temperatures up to $1400{ }^{\circ} \mathrm{C}$ until the sample just melted (approximately 1 minute). The IR spectra of the three different olivine particles are shown in fig. 1.

\section{An application to comet Halley}

The above mentioned laboratory data have been used to fit the $8-13 \mu m$ spectrum of comet Halley. 


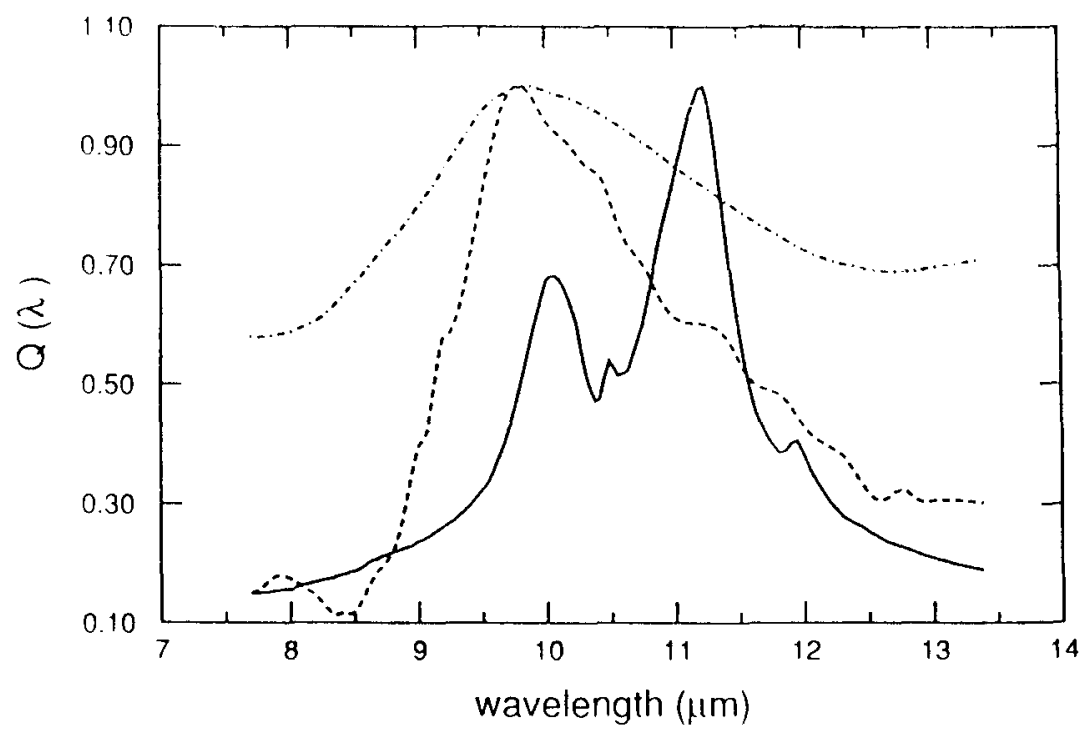

Fig. 1. Extinction efficiencies of the three different types of olivine analyzed in the present work: crystalline (solid line), amorphous (dashed line) and synthetic (dash-dotted line). The curves are normalized at their maximum.

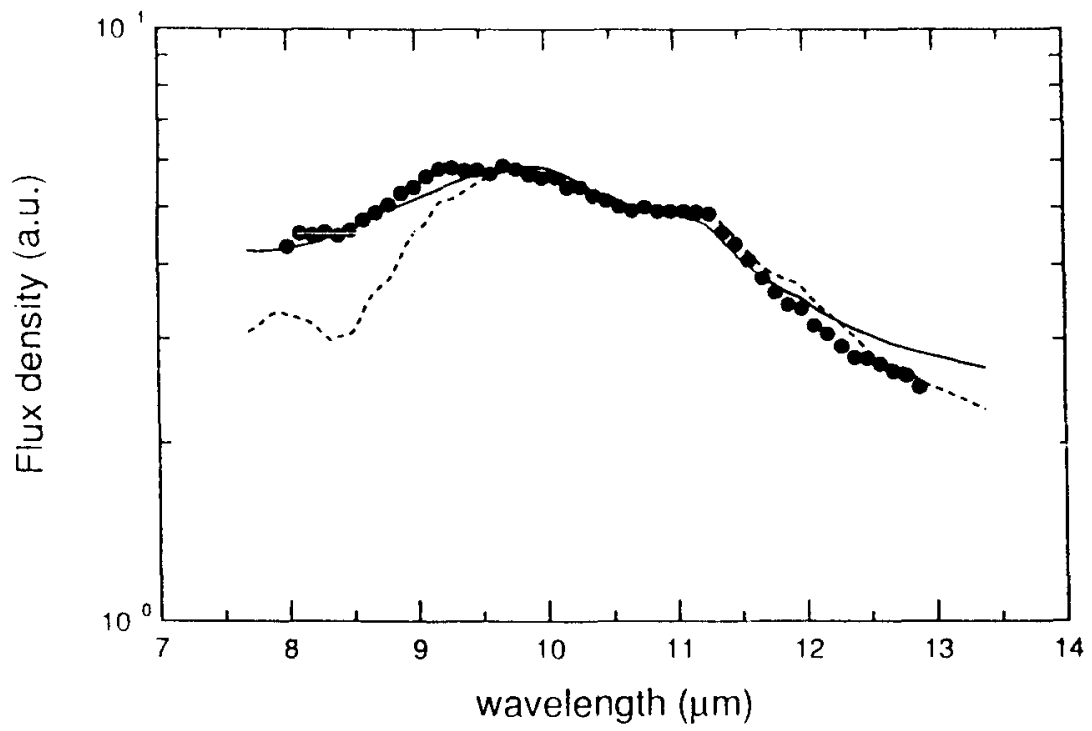

Fig. 2. Best fit of the observed (Campins and Ryan, 1989) Halley spectrum (dots) with laboratory data. The dashed curve is obtained for a $50 \%$ mixture of amorphous and crystalline olivine (best fit parameters are: $T_{n}=320 \mathrm{~K}, T_{d}=380 \mathrm{~K}, R_{d} / R_{n}=1000, A_{c}=1.086 \mathrm{~T}=0.5 \mathrm{mag}$ ). The solid curve is obtained by using synthetic olivine with only $5 \%$ of crystalline olivine. Best fit parameters are the same except for $A_{c}=0.25$ mag. 
The model we used to fit the cometary flux is an adaptation of that originally applied to circumstellar envelopes (Orofino et al., 1987; Blanco et al., 1989). The free parameters are: radius and temperature of the nucleus $\left(R_{n}, T_{n}\right)$, radius and temperature of the dust envelope $\left(R_{d}, T_{d}\right)$ and the total optical thickness $(\tau)$ of the dust at $8 \mu \mathrm{m}$. The values of some of these parameters are already available in literature. From our preliminary results, shown in fig. 2, it seems that the Halley $10 \mu \mathrm{m}$ feature can be satisfactorily fitted by a mixture of amorphous and crystalline olivine. We note however that a better fit is obtained by using the synthetic olivine with cosmic elemental composition. Moreover, in this case, a far smaller amount of crystalline component is needed. This result was also obtained by Greenberg (1990) and can be very important in the study of cometary evolution and its connections with interplanetary dust particles (Campins and Ryan, 1989; Sandford, 1989). It is worthwhile to note that laboratory IR spectra of crystalline olivine are usually taken by embedding dust samples in a matrix. This experimental procedure may change both the shape and the position of spectral features (Bohren and Huffman, 1983). At present extended laboratory work is in progress to account for this effect.

ACKNOWLEDGEMENTS. This work was partially supported by ASI, CNR-GIFCO and MPI. J. R. Stephens gratefully acknowledges the financial support of a NATO Collaborative Fellowship.

\section{REFERENCES}

Blanco, A., Borghesi, A., Bussoletti, E., Colangeli, L., Fonti, S., and Orofino, V. (1988) 'Amorphous carbon and silicon carbide grains around carbon stars', in E. Bussoletti, C. Fusco, and G.

Longo (eds.), Experiments on Cosmic Dust Analogues, Kluwer Academic Publishers, Dordrecht, pp. 167-173.

Bohren, C. F., and Huffmann, D. R. (1983) Absorption and Scattering of Light by Small Particles, Wiley, New York.

Bregman, J. D., Campins, H., Witteborn, F. C., Wooden, D. H., Rank, D. M., Allamandola, L. J., Cohen, M., and Tielens A. G. G. M. (1987) 'Airborne and groundbased spectrophotometry of comet $\mathrm{P} /$ Halley from 5-13 $\mu \mathrm{m}^{\prime}$ Astron. Astrophys. 187, 616-620.

Campins, H., and Ryan, E. V. (1989) 'The identification of crystalline olivine in cometary silicates' Astrophys. J. 341, 1059-1066.

Greenberg, J. M. (1990) private communication.

Knacke, R. F. (1989) 'Comet dust: connections with interstellar dust' in L. J. Allamandola and A. G. M. Tielens (eds.), Interstellar Dust, Kluwer Academic Publishers, Dordrecht pp. 415-428.

Kratschmer, W., and Huffman, D. R. (1979) 'Infrared extinction of heavy irradiated and amorphous olivine, with application to interstellar dust' Astrophys. Space Sci. 61, 195-203.

Orofino, V., Colangeli, L., Bussoletti, E., and Strafella, F.(1987) 'Amorphous carbon around carbon stars' Astrophys. Space Sci. 138, 127-140.

Sandford, S. A. (1989) 'Interstellar dust in collected interplanetary dust particles' in L. J. Allamandola and A. G. G. M. Tielens (eds.), Interstellar Dust, Kluwer Academic Publishers, Dordrecht pp. 403-413.

Stephens, J. R. (1980) 'Visible and ultraviolet $(800-130 \mathrm{~nm})$ extinction of vapor condensed silicate, carbon and silicon carbide smokes and the interstellar extinction curve' Astrophys. J. 237, 450-461.

Stephens, J. R., Blanco, A., Borghesi, A., Fonti, S., and Bussoletti, E. (1989) 'Infrared spectra of crystalline and glassy silicates and application to interstellar dust' in A. G. G. M. Tielens and L. J. Allamandola (eds.) Interstellar Dust Contributed Papers, NASA CP-3036, pp. 375-380. 